\title{
Lernräume für Intrapreneurship. Eine praxistheoretische Perspektive auf Grenzziehung und Grenzbearbeitung im Spannungsfeld zwischen Arbeitsalltag und Innovationsentwicklung
}

\author{
Andreas Schröer · Joy Rosenow-Gerhard $D$
}

Eingegangen: 4. Januar 2019 / Angenommen: 12. April 2019 / Online publiziert: 10. Mai 2019

(C) Der/die Autor(en) 2019

Zusammenfassung Im vorliegenden Beitrag untersuchen die Autoren die Konstituierung und Bearbeitung von Grenzen zwischen Arbeitsalltag und Innovationsentwicklung anhand eines Innovationslabors in der Sozialwirtschaft. Intrapreneure eignen sich dort unternehmerische Praxen an, um auf diese Weise soziale Innovationen $\mathrm{zu}$ fördern. Grenzziehung und -bearbeitung lassen sich deutlich machen an der räumlichen Strukturierung der performativen Praxis, der Konstitution eines symbolischen Raums, einem Rhythmus im Wechsel von Divergenz und Konvergenz, sowie Be- und Entschleunigung. Die Synchronisation des datenzeitlichen Rhythmus in der Prozessplanung und dem modalzeitlichen Rhythmus des Lernprozesses in den Teams ist eine zentrale Herausforderung (auch) für Laborkonzepte.

Schlüsselwörter Relationaler Raum · Soziale Innovation · Innovationslabor · Grenzziehung · Grenzbearbeitung · Lernen Erwachsener

\section{Learning spaces for intrapreneurship. A practice-theoretical perspective on the processes of boundary setting within the tug of war between everyday work routine and developing innovation}

\begin{abstract}
In this paper, the authors examine the setting of and working with boundaries between everyday work and innovation development, based on an empirical analysis of an innovation lab in the social economy. The lab allows intrapreneurs to learn and use entrepreneurial practices to foster social innovation. The process of boundaries setting can be made clear by the spatial structuring of performative
\end{abstract}

Prof. Dr. A. Schröer · Dipl.-Päd. J. Rosenow-Gerhard ( $\varangle)$

Universität Trier, Trier, Deutschland

E-Mail: joy.rosenow-gerhard@uni-trier.de

Prof. Dr. A. Schröer

E-Mail: schroeer@uni-trier.de 
practice, the constitution of symbolic spaces, the rhythm of divergence and convergence as well as acceleration and deceleration. The synchronization between the "data time" (Prange) of the process and the modal time of the participants is another crucial challenge for laboratory concepts.

Keywords Relational model of space - Social innovation - Innovation lab · Boundary setting $\cdot$ Working with boundaries · Adult learning

\section{Einleitung}

Die Fähigkeit zum unternehmerischen Handeln wird zunehmend im Bildungsbereich (Lackéus 2015) und in den sozialen Diensten (Nandan et al. 2015) als erforderlich angesehen, um mit dem schnellen Wandel veränderter politischer, ökonomischer und gesellschaftlicher Rahmenbedingungen angemessen umgehen zu können. Für das Erlernen unternehmerischer Fähigkeiten gelten erfahrungsbasierte und praxisorientierte Lernarrangements als geeignet (Deakins und Freel 1998), mit Anleihen an Ansätze problembasierten Lernens (Jones und English 2004). Entrepreneurship Education ist zudem Topos der Wissenschaftlichen Weiterbildung geworden.

Als „Unternehmerinnen“ und „Unternehmer“ werden Akteure bezeichnet, die Innovationen maßgeblich entwickeln, umsetzen und verbreiten (Swedberg 2009). Theorien zu sozialen Innovationen (Mulgan 2012) betonen, dass sich Innovationen durch neue Handlungsformen und -muster auszeichnen, die Verbreitung gefunden haben. Seit einiger Zeit gewinnen Innovationslabore an Bedeutung, um (soziale) Innovationen zu entwickeln und testweise umzusetzen (Gryszkiewicz et al. 2016). Solche Labore sind nicht nur Forschungs- und Entwicklungseinheiten, sondern auch Lernorte der Weiterbildung (Schröer und Schmitz 2019). Die Teilnehmenden (TN) werden unterstützt, unternehmerische Denkweisen anzunehmen und Fähigkeiten zu erwerben, die als unternehmerisch bezeichnet werden (Bacigalupo et al. 2016). Wenn es sich bei den TN um Intrapreneure handelt, also unternehmerisch handelnde Mitarbeitende in existierenden Unternehmen, wird die Vereinbarkeit von Arbeitsalltag und Laborteilnahme zur Gelingensbedingung des Innovationsvorhabens. Dies gilt besonders, weil Arbeitsalltag und Innovationslabor unterschiedlichen handlungsleitenden Logiken folgen, die March (1991) als ,exploitation“ (Alltag) und ,exploration“ (Labor) charakterisierte. Exploratives Lernen und Innovationsentwicklung sind demnach auf ein gewisses Maß der Abgrenzung vom Arbeitsalltag angewiesen, der die unmittelbare Verwertung des Angeeigneten verlangt.

Labore für Intrapreneurship lassen sich als temporäre Lernorte der Weiterbildung verstehen, insofern sie unternehmerisches Lernen von Individuen (pädagogisch gestaltet) unterstützen. Primär gelten sie zwar als Entwicklungsräume, in denen Innovationen für Organisationen erarbeiten werden. Die Reflexion auf Innovationslabore als noch wenig untersuchter Lernkontext ist jedoch erziehungswissenschaftlich attraktiv, weil darin individuelle und organisationale Lernebenen intendiert sind. Für beide Lernebenen ist das Verhältnis von Labor und entsendender Organisation zentral - für die TN im Sinne der Herausforderung der Vereinbarkeit von Arbeitsalltag und Teilnahme ebenso wie für TN in der Erwachsenenbildung (Schmidt-Lauff 2018). 
Für die Organisation steht die Durchlässigkeit von Organisationsgrenzen in Hinblick auf innovationsorientierte Praktiken und entwickelte Lösungen im Vordergrund.

Aus pädagogischem Interesse an der lernunterstützenden Funktion des Labors werden im zugrunde liegenden Projekt nicht die Praktiken des Lernens fokussiert, sondern die beobachtbare Lernunterstützungspraxis. Dabei unterscheiden wir zwischen der angebotenen und der von den TN angenommenen Lernunterstützung. Für die Lernunterstützung ist das Verhältnis von Arbeitsalltag und Laborteilnahme und die raumzeitliche Konstitution der Grenzen zwischen beiden genauer zu analysieren (Schröer und Händel 2019). Das soziale Handeln der TN (re-)produziert Raum(an)ordnungen (Löw 2001), andererseits strukturieren diese z. T. symbolischen Ordnungen das Handeln der TN. An diesem - in der Erwachsenenbildung bekannten - doppelten Verweiszusammenhang von Struktur und Handlung (Kraus et al. 2016) knüpft unsere Analyse der lernunterstützenden Wirkung von Raumordnungen an.

Wie konstituieren sich die Grenzen eines Labors für Intrapreneurship zum Arbeitsalltag? Wie bearbeiten TN diese Grenzen, um sie produktiv werden zu lassen? Diesen Fragen geht der vorliegende Beitrag in praxistheoretischer Perspektive nach. In dieser Perspektive wird das Verständnis von Grenzen und Grenzziehung eines relationalen Raums (Löw 2001) mit der zeittheoretischen Grundlegung der Synchronisierung von Datenzeit (Verlaufszeit) und Modalzeit (Eigenzeit) (Berdelmann 2010) verschränkt. Dadurch wird der Umgang der TN mit den Grenzen des Labors in raumzeitlicher Perspektive deutlich. Für den vorliegenden Text haben wir den Schwerpunkt auf die räumliche Betrachtung gelegt und insbesondere Material an den Stellen analysiert, wo die räumliche und die zeitliche Perspektive zusammenkommen.

Im Folgenden leiten wir nach einer theoretischen Einführung zu drei gegenstandsbezogenen Fragestellungen über, welche anschließend die Struktur unserer Ergebnisdarstellung bieten. Wir schließen mit der zentralen Herausforderung und Desiderata zur weiteren Analyse.

\section{Theoretischer Rahmen und Entwicklung der Fragestellungen}

Ausgehend vom Practice Turn in den Sozialwissenschaften (Schatzki 2001) verstehen wir Organisationen, und damit die Organisation Labor, als Bündel von Praktiken und materialen Arrangements. Praktiken werden als raum-zeitlich strukturiert gefasst, sie beinhalteten Know-how, anleitende Regeln und eine teleologisch-affektive Struktur (Ziele, Emotionen). Für deren Analyse ist ein Verständnis des Kontextes ebenso wichtig wie die Arrangements aus materiellen Objekten, wie Personen, Artefakten, Organismen und Dingen. Praktiken in Organisationen geschehen ereignishaft, ähnlich wie in einer Aufführung, sie werden in performativen, raum-zeitlich strukturierten Akten aktualisiert (Reckwitz 2002).

Im Fokus des Forschungsvorhabens steht ein Innovationslabor, das von mehreren Sozialunternehmen aus Diakonie und Caritas gemeinsam betrieben wird und darauf abzielt, durch Social Intrapreneurs (Schmitz und Schröer 2016) soziale Dienstleistungsinnovationen im Kontext dieser Sozialunternehmen hervorzubringen. Das Innovationslabor soll unternehmerisches Lernen unterstützen und kann daher als 
Organisationsgrenzen überschreitender temporärer Lernraum verstanden werden. In diesem pädagogischen Raum werden unternehmerische Praktiken eingeübt, die sich von den professionellen Praktiken in den Herkunftsorganisationen der TN (hier Sozialunternehmen und Wohlfahrtsverbände) unterscheiden. Im Folgenden werden Praktiken der Konstitution, Bearbeitung und Reflexion dieser Grenze zwischen Labor und Herkunftsorganisation analysiert.

Den Raum des Labors verstehen wir als relationale (An-)Ordnung von Menschen und sozialen Gütern (Spacing), der von Menschen durch kontinuierliche Platzierung und Verknüpfung konstituiert und auf symbolischer Ebene durch die integrativen Interpretationsleistungen von Menschen synthetisiert wird (Löw 2001). Grenzziehung entsteht zunächst durch die örtliche Separierung der Laborarbeit vom Arbeitsalltag, d.h. das Labor wurde in einem kommunalen Innovation Hub eingerichtet. Zur Konstituierung gehören die Andersartigkeit der örtlichen Settings im Labor und die räumliche Strukturierung der Innovationspraktiken, die wiederum zur Entstehung symbolischen Raumes beitragen. Praktiken des Verweisens, Zeigens oder Implementierens der prozessverantwortlichen Labormoderatorinnen sind dabei konstitutives Merkmal pädagogischer Räume (Göhlich 1993). Die lernunterstützende Qualität von Räumen wird im Wechselspiel von Öffnung und Schließung des pädagogischen Raums zur Welt; hier etwa die Welt der entsendenden Organisation oder der Alltag der späteren Nutzerinnen der neuen sozialen Dienstleistungen deutlich. Diese Überlegungen führen zur ersten forschungsleitenden Frage für die folgende empirische Analyse: Inwieweit unterstützt die besondere räumliche Strukturierung der performativen Praxis im Labor das unternehmerische Lernen der Teilnehmenden (z. B. durch Öffnung und Schließung des Labors zur äußeren Welt)?

Das Intrapreneurship-Labor wurde als ein partiell handlungsentlasteter Raum (,,Schonraum“) konzipiert, in dem die TN in Abgrenzung zu ihren sonstigen Alltagsanforderungen neue Lösungsideen entwickeln können. Trotz thematischer Anknüpfungspunkte an die Arbeit in den Herkunftsorganisationen soll das Labor als separater Raum die Konzentration auf Entwicklung und Lernen ermöglichen. Zugleich sollen die Grenzen zu den entsendenden Unternehmen so weit transparent sein, dass es nicht zu allzu großen Fremdheits- oder Abstoßungsreaktionen zu den entwickelten Lösungen kommt. Das Labor ist dabei mehr als ein metaphorischer Schonraum, denn es handelt sich um einen physischen Ort, der jedoch auch symbolisch gefüllt wird. Für diese symbolische Ebene verwenden wir in der zweiten forschungsleitenden Fragestellung den Begriff des symbolischen Raums: Wie vollzieht sich die Kreation symbolischen Raumes im Labor durch spezifische Praktiken und performative Akte der Beteiligten?

Die Grenzkonstitution wird durch temporäre Muster unterstützt, sodass sich die zeitliche und räumliche Ebene grenzkonstituierender Praktiken verschränken. Zur zeitanalytischen Einordnung ist zunächst auf die elementare Bedeutung von Zeit, Entwicklungsphasen und Rhythmus in der Innovationsforschung zu verweisen. In Innovationsprozessen spielen chronologische Strukturierungen in Entwicklungsphasen eine wichtige Rolle (z. B. Invention, Innovation und Diffusion; vgl. Schumpeter 1942). So wird im Sinne der Innovationsförderung und kreativer Problemlösung vor allem Wert gelegt auf die Rhythmisierung (Zaltman et al. 1973) von Phasen der Divergenz und Konvergenz (Rickards 1994). Vergleichbar mit der erziehungswis- 
senschaftlichen Analyse des Unterrichtsgeschehens soll in methodisch unterstützten Innovationsprozessen das Potential oszillierender Wechsel (Berdelmann 2010) zwischen varianzsuchenden und -reduzierenden Phasen ausgeschöpft werden. Auch Tempowechsel und Geschwindigkeit markieren eine Differenz zum Arbeitsalltag, in dem Lösungen (Projekte) über längere Zeiträume entwickelt und Entscheidungen (z.B. welche Idee verfolgt wird) nicht so schnell getroffen werden wie im Labor. Interessant ist dabei insbesondere die Synchronisation von didaktischer Konzeption (Datenzeit) und der Eigenzeit der Lernenden (Modalzeit) (Prange 2009). Die dritte forschungsleitende Frage lautet daher: Inwieweit unterstützt der Rhythmus im oszillierenden Wechsel zwischen Phasen der Divergenz und Konvergenz, zwischen hoher Geschwindigkeit und Entschleunigung der Konstitution labortypischer zeitlich-strukturierter Innovationspraktiken?

Diese drei Leitfragen orientieren die empirische Analyse, deren methodisches Vorgehen im Folgenden dargestellt wird.

\section{Methodisches Vorgehen}

Das Material entstammt einem Forschungsprojekt über ein Innovationslabor, das mehrere Sozialunternehmen gemeinsam betreiben. In der Datenerhebung kamen insbesondere die Teilnehmende Beobachtung jedes Workshops (WS) und Interviews zu zwei Zeitpunkten zum Einsatz. Die Teilnehmende Beobachtung wurde jeweils von einem Mitglied des Forschungsteams während der einzelnen sechs Workshops (WS) durchgeführt. Die Beobachtende nahm am gesamten WS teil, ohne jedoch Prozessschritte selbst mitzuvollziehen. Interaktion beschränkte sich auf Situationen, in denen die Forscherin selbst angesprochen und einbezogen wurde, und schloss eine einflussnehmende Kommunikation aus. Die Themenfelder des Beobachtungskatalogs deckten die Bereiche „Setting“, „Adressierweisen“, „Formulierte Ziele des Labors“, „Methodeneinsatz“, „Verständnis von Unternehmertum“, „Lernpraxen“, „Zusammenarbeit und Interaktion in und zwischen Teams“, „Bedingungen für Intrapreneurship“ und die „Projektideen“ ab. Die leitfadengestützten Interviews mit den $T N$ zu zwei Zeitpunkten dienten der Generierung von Erzählungen und der Evaluation zu den Themenblöcken „Motivation und Ziele“, „Selbsteinschätzung in Bezug auf unternehmerische Fähigkeiten“ und „Erwartungen an die Teilnahme am Labor“ bzw. im Abschlussinterview der „Erwartungserfüllung“, ,Vereinbarkeit der Teilnahme mit dem Alltag“, ,Zusammenarbeit im Team/Gruppe/mit den Moderatorinnen/ Experten“ sowie „Veränderungen der eigenen Person und Lernzuwachs“. Die Daten wurden telefonisch erhoben (t1 vor WS2, t2 nach WS6; $n=24)$ und anschließend transkribiert. Alle Daten wurden in Anlehnung an die Qualitative Inhaltsanalyse nach Mayring (2010) und in Anlehnung an die Praxeography nach Bueger (2013) codiert und verschränkt in mehreren Durchgängen ausgewertet. ${ }^{1}$

\footnotetext{
1 Darüber hinaus kamen im Forschungsprojekt weitere Interviews mit den entsendenden Vorgesetzten und Moderatorinnen im Labor, sowie Dokumentenanalyse und weitere Audio-, Foto- und Videoaufzeichnungen zum Einsatz, die jedoch aufgrund des aktuellen Projektstands (laufende zweiten Erhebungsphase) nicht in die vorliegende Analyse einbezogen werden konnten.
} 


\section{Ergebnisse der empirischen Untersuchung}

Der Laborprozess setzte sich zusammen aus sechs Präsenzworkshop-Tagen und konkreten Arbeitsaufgaben für die Zwischenzeiten. Zusätzlich fanden eine Auftakt-, Abschluss- und Expertenveranstaltung statt, zu der Vertretende der entsendenden Organisation (Vorgesetzte, Kolleginnen), Expertinnen sowie die interessierte Öffentlichkeit eingeladen wurden. Im Labor wurden praxiserprobte Methoden zur Entwicklung innovativer Dienstleistungen, wie Human Centred Design, Business Model Generation (Osterwalder und Pigneur 2010), und die Herangehensweise des Lean Startup (Ries 2011) kombiniert. Die Orientierung an den Bedarfen der späteren Nutzergruppen war dabei zentral für die Entwicklung der angestrebten Geschäftsmodelle. Zwischen den WS arbeiteten die TN individuell an ihren Projekten, konnten jedoch jederzeit Beratung von den beiden Moderatorinnen oder Experten in Anspruch nehmen.

In Bezug auf die forschungsleitenden Fragen konnte Folgendes exemplarisch festgestellt werden.

\subsection{Inwiefern unterstützt die besondere räumliche Strukturierung der performativen Praxis im Labor das unternehmerische Lernen der Teilnehmenden?}

In Innovationslaboren, in denen Design Thinking (Plattner et al. 2009) zum Einsatz kommt, wird in der Regel ein hohes Maß an räumlicher Flexibilität benötigt, um unterschiedliche Konstellationen der Team- und Gruppenarbeit zu ermöglichen. Dies zeigt sich z. B. in mobilen Sitzmöbeln und beweglichen Elementen, wie Pinnwänden. Hieran zeigt sich eine konzeptionelle Differenz zu einem durchschnittlichen Büro, in dem üblicherweise Möbel wie Schreibtische, Regale, Schränke und Tische nicht beweglich sind, ebenso wie die Differenz zu einem gewöhnlichen Seminarraum mit Tischen und Stühlen mit der üblichen Ausrichtung auf eine Projektionsfläche.

Auffällig ist, dass die Veranstaltungsräume in der vorliegenden Untersuchung eine konzentrische räumliche Standard-Konfiguration aufweisen, die bei aller potenziellen Flexibilität des Raumes und der Methode nicht verändert wurde: Die Stühle sind im Kreis ausgerichtet und dahinter die Pinnwände aufgestellt, eine pro Zweierteam, einige Tische sind an den Rand geschoben. WS 1, 2 und 5 finden in einem kleineren Raum (49 qm) statt, in dem sich die TN einfach auf ihrem Stuhl umdrehen, um am Tisch dahinter zu arbeiten. Der größere Raum (WS 3, 4, 6) weist dasselbe Arrangement auf einer größeren Fläche (140 qm) auf. Diese Standard-Konfiguration bleibt auch in der Nutzung der TN nahezu unverändert, die angebotene Flexibilität des räumlichen Arrangements wird nur im Ansatz genutzt: In WS1 nehmen einige TN ihre Pinnwand zu einem anderen Ort mit; in allen anderen WS arrangieren weder TN noch Moderatorinnen den Raum um.

Beispiel: Zwei TN eines Teams drehen sich in WS1 auf ihrem Stuhl um und verwenden die Pinnwand hinter ihnen. Sie stehen nach kurzer Zeit auf und arbeiten stehend im Bereich zwischen Stuhllehne und Pinnwand, verändern jedoch keins der beiden, sodass der Abstand von weniger als $1 \mathrm{~m}$ nicht variiert wird (die Platzverhältnisse hätten dies ermöglicht). 
Die TN melden im Abschluss-Feedback zurück, dass sie das hohe Maß an Flexibilität in der Workshop-Gestaltung sehr geschätzt hätten. Aus Sicht der Teilnehmenden Beobachtung lässt sich diese Flexibilität zwar bezogen auf die zeitliche Strukturierung bestätigen, nicht jedoch im Hinblick auf die räumliche Konfiguration. Die räumliche Stabilität scheint den Bedürfnissen der TN zu entsprechen, die bei der Beschreibung ihrer optimalen Lernräume teilweise ihre Büros beschreiben, welche z.B. über einen sehr aufgeräumten Schreibtisch verfügen. Anstelle der methodisch vorgesehenen Differenz zwischen stabiler räumlicher Struktur im Arbeitsalltag und Flexibilität in den Laborphasen wird der Stabilität des Arbeitsalltags eine weitere stabile Konfiguration (Labor) gegenübergestellt. Durch diese beiden stabilen räumlichen Konfigurationen wird die methodisch intendierte Grenze zwischen Labor und Arbeitsalltag (Stabilität - Flexibilität) erodiert.

Eine weitere Differenz wird im Modus der Zusammenarbeit deutlich. Während im Labor mehrere Ebenen der Zusammenarbeit (Zweierteam, Team-Tandems, Großgruppe) vorgesehen sind, arbeiten einige TN im Alltag eher alleine (z. B. Stabsstelle), andere haben keinen täglichen Kontakt zu Kolleginnen, da sie überwiegend im Homeoffice arbeiten. Die Arbeit im Team markiert demnach eine Grenze zum Arbeitsalltag. In den Eingangsinterviews äußern einige TN, dass sie sich sehr auf die enge Zusammenarbeit mit einer Teampartnerin bzw. -partner freuen. Im Abschlussinterview wird deutlich, dass die enge Zusammenarbeit im Zweierteam als förderliche Komponente für den eigenen Lernprozess und die Entwicklung des Geschäftsmodells (Perspektivwechsel, Arbeitsteilung, fachliche Ergänzung) wahrgenommen wurde.

Auffällig ist, wie stark die räumliche Konfiguration auf die Zusammenarbeit im Zweierteam als Kernarbeitseinheit ausgelegt blieb (die Teamkonstellation wird nur in einer Warm-up-Übung in WS2 aufgelöst, in zwei Situationen wurden je zwei Teams für eine Feedbacksituation gematcht). Dies steht im Gegensatz zur Betonung von Multiprofessionalität und Diversität im methodischen Ansatz. Die räumliche Konfiguration beschränkt hier die Möglichkeiten design-orientierter Innovationsentwicklung.

Grundsätzlich artikulieren die TN die Wichtigkeit des örtlichen Abstands, der damit als lernunterstützendes Element gesehen wird:

Es war wichtig, dass wir uns die Zeit genommen haben. [...] weil wenn man jetzt da im Büro sitzt oder so und ähm sowas nebenher versucht zu erledigen, dann ist das nicht möglich, also das, das war schon gut, [...] weg von der gewohnten Umgebung und einfach hin ähm in was Neues, also. Meistens kommen dann auch neue Gedanken, wo man vorher nicht dran gedacht hat. Wenn man sich nicht dort befindet, wo man sich sonst eh aufgehalten hätte. (TN2, t2).

Den TN gelingt es, die Grenze produktiv zu bearbeiten und lernunterstützend zu nutzen: Fast alle Teams haben eine mehrstündige Hin- und Rückfahrt mit Auto oder Bahn, während der bereits viel besprochen und an den Projekten weitergearbeitet wird. Andererseits scheint auch die Distanz zu begrenzen, da ein Entsender im Abschlussinterview davon spricht, dass er der Einladung zur Runde der Expertinnen und Experten zwar einmal gefolgt ist, aber die örtliche Distanz einfach zu weit für einen weiteren Besuch gewesen sei. 


\subsection{Wie vollzieht sich das Synthetisieren des symbolischen Raumes durch spezifische Praktiken und performative Akte der Beteiligten?}

Im Folgenden wird gezeigt, welche symbolischen Räume die TN hervorbringen und damit das Labor positionieren. Das Labor erfüllt aus Sicht der TN verschiedene Funktionen, die dessen Abgrenzung zum Arbeitsalltag deutlich machen. Beispielsweise möchte ein TN das Labor explizit als Lernraum nutzen. Im Eingangsinterview zählt er Dinge auf, die er explizit lernen möchte und erläutert „Das sind Fähigkeiten, die ja sonst in meinem Alltag auch nicht geschult werden" (TN1, t1). Auch eine andere TN beschreibt schon vorab sie habe ,immer das Gefühl, dass ich nicht gut genug äh sprechen und mich darstellen kann in wichtigen Situationen, und da erhoffe ich für mich persönlich einfach einige Tipps. Besser auf'n Punkt zu kommen zum Beispiel, solche Dinge“ (TN4, t1).

Das Labor wird auch als Schonraum wahrgenommen: TN10 bezeichnet das Labor im Interview (t2) als „Schutzblase“. Auch die Moderatorinnen verweisen auf den partiell handlungsentlasteten Schonraum: „Wenn ihr zurück geht an eure normalen Arbeitsplätze habt ihr noch andere Aufgaben" (W3, WS1). Auf der anderen Seite berichten fast alle $\mathrm{TN}$, dass es eine intensive und anstrengende Zeit war, die sie auch herausgefordert hat, beispielsweise durch die Pitches und das Präsentieren vor fremden Menschen.

In Bezug auf die Positionierung gegenüber Dritten stellt TN6 den Vergleich mit der Fernsehsendung „Höhle der Löwen“ als sehr passend für die Beschreibung des Labors vor. Sie berichtet auch davon, dass ihre Kollegen ihre Teilnahme am Labor als ,Wellness-Fortbildung“ bezeichnen würden, da sie und ihr Teamkollege „,immer so gut gestimmt zurückgekommen“ seien (TN6, t2). Die TN legitimiert die eigene Teilnahme mit der um Dramaturgie und Spannung bemühten TV-Sendung und wertet diese damit auf bzw. grenzt sie gleichzeitig von „Wellness“ ab.

Das Labor als Experimentierraum wird insbesondere anhand der verschiedenen Materialien, die permanent zur Verfügung stehen, wahrgenommen. Einen Schwerpunkt des Design Thinking bilden die Visualisierung aller Gedanken und Notizen sowie das bildliche Gestalten eines Prototyps. Mit Klebezetteln in verschiedenen Farben und Größen können Gedanken festgehalten und jederzeit neu arrangiert werden. Insbesondere in WS2 kommen Kreativtechniken zum Einsatz, denn die TN sollen aus ihrer ersten Ideengenerierung zwei Ideen auswählen und diese mit Hilfe eines Prototyps veranschaulichen. Dafür wird unterschiedliches Material in der Mitte des Stuhlkreises zur Verfügung gestellt (Lego-Bausteine, Bastelmaterial, vorgefertigte Szenen, Figuren, Sprechblasen etc.). Die TN bedienen sich sofort und erstellen kreative Prototypen ihrer Lösungsansätze. Dies ist der körperlich aktivste WS, wo zwar die örtliche Konfiguration nur minimal angepasst wird, aber die TN immer wieder von ihren Plätzen zum Materiallager in der Mitte gehen und zeitweise auf dem Boden sitzend oder hockend arbeiten. Positiv bewerten die TN, dass im Hinblick auf die Materialien ,,immer alles da“ gewesen sei, sodass Kreativität entstehen konnte. Die TN verwenden je Gruppe wenig, insgesamt jedoch von allen vorhandenen unterschiedlichen Materialientypen.

Des Weiteren wird das Labor von den TN als Reflexionsraum charakterisiert. Anhand einer Lebenslaufanalyse in WS1 wurde der Blick zunächst retrospektiv auf die 
persönlichen Ressourcen, anschließend auf gemeinsame Ressourcen und Netzwerke des Teams gelenkt. Obwohl dieser Rückblick nur insgesamt wenige Minuten lang war wird das Lab als Reflexionsraum wahrgenommen. Ein TN gibt darüber hinaus an, dass das Lab für ihn insgesamt ein „Raum zum Denken“ (TN2, t2) war. Gegenüber Dritten beschreibt er daher: ,Wir haben eine Idee und dort haben wir die Zeit und den Raum das zu vollenden und durchdenken, einfach um frei denken zu können“" (TN2, t2).

Zudem konstituiert sich der symbolische Raum als Bühne. Elevator Pitches sind in der Start-Up-Szene ein gängiges Instrument, um in kurzer Zeit interessierten Personen den persönlichen Lösungsansatz für das Problem einer (sozialen) Bedarfsgruppe nahe zu bringen. Speziell bei der Suche nach Investoren haben sich mittlerweile unterschiedliche Formen etabliert. In jedem WS wird mindestens einmal gepitcht und von den anderen TN und Moderatorinnen Feedback gegeben. In WS3 waren stundenweise Expertinnen, Vorgesetzte und Kolleginnen der TN eingeladen um zur Idee in einem frühen Stadium Feedback einzuholen. In WS6 wurden so auch die Ergebnisse vorgestellt.

Bei der Einübung der Pitches wurde das Labor in eine Vorder- und Hinterbühne arrangiert: Die WS1, 2 und 5 finden im kleineren Seminarraum statt, neben dem ein Arbeitsraum mit vielen unterschiedlichen Sitz- und Arbeitsmöbeln liegt. In den Arbeitsphasen ziehen sich einige Teams dorthin zurück. Anschließend wird das Ergebnis in Form eines dreiminütigen Pitches aufgeführt. Dabei variiert, ob die Teams für den Pitch (mit Pinnwand) in den Seminarraum zurückkehren oder ob die anderen TN zu ihrer Pinnwand kommen. Letzteres bedeutet, dass die gesamte Gruppe den eigentlichen Seminarraum verlässt. Im größeren Seminarraum sind die Pinnwände um den Stuhlkreis herum aufgestellt, der Raumwechsel entfällt. Die zeitliche Rhythmisierung ist klar und wird von den TN eingehalten: Während je ein Team präsentiert notieren die anderen Stichworte auf einem Klebezettelblock. Der Wecker beendet die Performanz der Projektpräsentation. Die anderen TN geben nun (anhand eines Schemas) Feedback und reichen ihre Notizzettel an die Präsentierenden, welche die Zettel an die Pinnwand kleben. Rhythmus und Dauer sind allen bekannt und grundsätzlich jedes Mal gleich. Die örtliche Trennung unterstützt den Aufführungscharakter. In WS3 und WS6 spendet die erweiterte Zuhörerschaft Applaus, was den Aufführungscharakter unterstützt und formalisiert.

\subsection{Inwieweit trägt der Rhythmus im oszillierenden Wechsel zur Konstitution labortypischer zeitlich-strukturierter Innovationspraktiken bei?}

Aus dem Diskurs der Innovationsförderung ist hinreichend bekannt, dass Innovationsentwicklung Zeit und Raum braucht. Dies liegt letztlich im Begründungshorizont der Innovationslabore verankert, die einen Raum abseits des Alltagsgeschäfts bieten um an Ideen zu arbeiten (Durst und Stahle 2013). Die zeitliche Rhythmisierung im Labor durch die Moderatorinnen stellt eine Differenz zum Arbeitsalltag der TN da. Die meisten TN berichten, dass sie im Arbeitsalltag relativ frei in ihrer Zeiteinteilung sind, sodass die enge zeitliche Taktung insbesondere in der ersten Hälfte einen groBen Unterschied darstellt. Durch die freie Zeiteinteilung hatten die überwiegenden TN daher eher selten Schwierigkeiten, die Vor- und Nachbereitung der WS in ihren 
Arbeitsalltag zu integrieren. Allerdings verfügten nur sehr wenige der TN über eine Freistellung von Aufgaben, sodass die Teilnahme am Labor (inkl. Vor- und Nachbereitung) zwar grundsätzlich Arbeitszeit war, jedoch zu den üblichen Aufgaben hinzukam.

Konzeptionell sind die WS klar gegliedert und in einen festen Ablauf gebracht, der auch den TN vorab vorliegt (Uhrzeit, Phasen und Aufgaben). Es fällt auf, dass nahezu jeder Tag anders verläuft als in dieser Agenda vorgesehen. Der einzige fixe Zeitpunkt scheint das Mittagessen zu sein (vermutlich aufgrund eines externen Caterers). Von dieser konzeptionellen Rhythmisierung der Datenzeit wurde in jedem WS abgewichen und Rhythmus und Inhalte dem geäußerten Bedarf der TN und somit den Eigenzeiten der Lernenden angepasst. Die Eigenzeit der Lernenden wurden zwar respektiert, die dadurch entstandenen Verzögerungen jedoch von Seite der TN als „Leerlaufzeiten“ (TN5, t2) interpretiert.

Auch an anderer Stelle wurde die Ausdehnung der Eigenzeit des Lernens durchaus akzeptiert: Ein Team hat bis zum Schluss keine Interviews mit der adressierten Zielgruppe geführt, weil die TN Zugangsschwierigkeiten hatten. Hier zeigt sich exemplarisch die zentrale pädagogische Herausforderung der Synchronisation im Spannungsverhältnis zwischen didaktischer Konzeption (,Datenzeit“) und der Eigenzeit der Lernenden (,Modalzeit“). Da die gegliederte Zeit geplanter Abläufe der Moderatorinnen der unverfügbaren Eigenlogik des Lernens der Intrapreneurs gegenübersteht, ist zwischen beiden Synchronisation erforderlich. Während die Datenzeit von einem Wechsel zwischen Phasen der Divergenz und Konvergenz ebenso wie von Phasen hoher Geschwindigkeit und Entschleunigung geprägt ist, lösen einzelne Teams diese Rhythmen in ihrer Modalzeit auf. Toleriert von der Moderation dehnen sie Phasen aus, oder schließen Prozessschritte nicht ab. Die Teilnehmenden Beobachtungen zeigen, dass der Abgleich zwischen Modal- und Datenzeit im Sinne einer Synchronisation häufig nicht aktiv vorgenommen wird.

Zum Laborprozess gehören Tempowechsel: Zu Beginn war der Rhythmus sehr schnell, die TN mussten viele kurze Aufgaben bearbeiten (Reflexion der Ressourcen, Ideation, Prototyping, Testing). Insbesondere während der Ideation wurde mit hoher Geschwindigkeit vorgegangen, während in anderen Phasen entschleunigt wurde. Die TN berichten überwiegend, dass sie die zeitliche enge Taktung zwar zu Beginn als Herausforderung, nach kurzer Zeit aber als unterstützend wahrgenommen hätten. In der zweiten Hälfte war der Rhythmus nicht mehr hoch und wurde als ,bissl viel Leerlauf“ (TN1, t2) oder ,bissl wie Kaugummi“" (TN2, t2) wahrgenommen.

Grenzbearbeitung zeigt sich u. a. im Umgang der Intrapreneure mit dem zeitlichen Umfang der Projekte, die sich nicht gänzlich innerhalb der Arbeitszeit bearbeiten lassen. Während alle TN für die WS freigestellt sind, lassen sich die umfängliche Vor- und Nachbereitung und die gedankliche Entwicklungsarbeit nur schwer auf Arbeitszeiten eingrenzen, wodurch automatisch Überschneidungen mit der Freizeit eintreten. Eine TN artikuliert dies im Zusammenhang mit ihren Kolleginnen und Kollegen, über die sie sagt: „Die denken man macht sich einen faulen Lenz, die sehen nicht was da für eine Arbeit drin steckt und was wir da auch für eine Freizeit reinstecken" (TN10, WS4). Während den TN die zeitliche Grenzziehung teilweise schwerfällt, fällt auf, dass Vorgesetzte zwar Mitarbeitende entsenden und ihnen so Raum und Zeit zur Verfügung stellen, selbst jedoch nicht alle bereit sind, Zeit für 
die Exploration im Labor zu investieren. In WS4 entsteht eine Diskussion, wie Erwartungen des organisationalen Umfelds und Projektstand besser in Einklang gebracht werden können. Eine TN antwortet „Die müssten alle mal hier her kommen, und nicht nur sagen ich hab keine Zeit“ (TN10, WS 4). ${ }^{2}$ Die TN markiert „hier“ die Grenze des Labors analog zum Wissensvorsprung, der mit der Teilnahme verbunden ist.

\section{Diskussion}

In der betrieblichen Wirklichkeit und der Startup Szene sind Innovationslabore verbreitet und aus Perspektive der Erwachsenenbildung als Lernorte von Interesse. Labore die sich primär an Intrapreneurs (hier: Mitarbeitende von Sozialunternehmen und Wohlfahrtsverbänden) richten, stehen dabei in einem Spannungsverhältnis zwischen dem Arbeitsalltag der entsendenden Organisation und dem raum-zeitlich verfassten, kreativ-kooperativem Lernarrangement des Labors.

Die empirische Analyse zeigt zunächst erwartungsgemäß, dass örtliche Arrangements zur Grenzkonstitution zwischen Labor und Herkunftsorganisation beitragen. Die räumliche Grenzziehung wird von TN als hilfreich und lernunterstützend wahrgenommen (materielle Bedingungen, Ausstattung, Zweierteams, Austausch mit den anderen TN und Moderatorinnen). Die untersuchten Formen der Grenzbearbeitung zwischen Labor und Herkunftsorganisationen zeigen räumliche (z.B. örtliche Dis$\operatorname{tanz}$ wird in Lernunterstützung umgewandelt) und zeitliche Ausprägungen (z.B. Anpassung des modalzeitlichen Rhythmus in der Datenzeit der TN) sowie solche auf Ebene der Praktiken (z. B. der Teamarbeit). Insbesondere die Rhythmisierung der Praktiken durch den zeitlichen Takt der Arbeitsschritte, die hohe Geschwindigkeit und enge zeitliche Vorgabe im Labor unterschied sich deutlich von der Rhythmisierung im Arbeitsalltag der Herkunftsorganisationen. Zur Synthetisierung trägt das Einüben bestimmter Praktiken bei, in denen eine Art innovationsorientierte bzw. unternehmerische Kultur zum Ausdruck kommt. Die TN erproben angebotene Praktiken und materiale Elemente (Struktur für Feedback, Struktur für Pitches, Wecker für Zeiteinhaltung usw.). Weniger deutliche Grenzen zeigten sich u. a. bei der Stabilität des örtlichen Arrangements. Zeitliche Übergänge zwischen Lernzeit und Freizeit wurden in der reflexiven Grenzbearbeitung einiger TN fließend wahrgenommen, ohne dass dies in den Herkunftsorganisationen entsprechend anerkannt war.

Neben der geografischen Trennung und räumlichen Strukturierung trägt in der Wahrnehmung der TN der symbolische Laborraum als Bühne, Schon-, Lern-, Experimentier- und z. T. auch Reflexionsraum zur Raumkonstitution in seiner Abgrenzung zum Arbeitsalltag bei. Dabei überlagern sich verschiedene Funktionen des symbolischen Raums, z.B. Motivation, Ideation, Präsentation, Exploration und Austausch (vgl. auch Jochumsen et al. 2014).

\footnotetext{
2 Für WS3 und die Abschlusspräsentation in WS6 wurden die Vorgesetzten und weitere Expertinnen und Experten für Feedback zum jeweiligen Projektstand ins Labor eingeladen. TN10 bezieht sich dabei auf die Vorgesetzten, die der Einladung nicht gefolgt sind.
} 
Nach außen wird das Labor legitimierend in Abgrenzung zum Organisationsalltag positioniert. Diese konzeptionelle Grenze wird durch die symbolische Raum-Ebene und die Einführung routinisierter Praxen, wie den Pitches, verstärkt.

Im kollektiven Zeiterleben des Labors wird die Eigenzeit des Lernens als besonders wertvoll und schützenswert wahrgenommen und markiert eine spezifische Diskontinuität zur zeitlichen Strukturierung der Praktiken des Arbeitsalltags (Hof et al. 2010). Die TN nutzen die dargebotene Struktur aus Divergenz und Konvergenz für ihre Projektentwicklung. Allerdings hat sich die Synchronisation von datenzeitlichem Rhythmus in der Prozessplanung und modalzeitlichem Rhythmus des Lernprozesses in den Teams als zentrale Herausforderung herauskristallisiert. Konkrete Formen der Synchronisation und Asynchronisation zu unterscheiden, haben wir daher als wichtige Aufgabe für die weitere Datenanalyse identifiziert. Darüber hinaus birgt die Analyse der produktiven Grenzbearbeitung dieser Praxisgemeinschaften Chancen für organisationales Lernen, welche an anderer Stelle vertieft analysiert werden sollen. Während die Lernunterstützung für die TN im Labor direkt beobachtbar war, ist die Unterstützung von Lernimpulsen an die entsendenden Organisationen nur indirekt zugänglich. Auch hierfür sind raum-zeitliche Arrangements und Laborgrenzen relevant. Durch die Teilnahme am Labor und die Aufrechterhaltung der symbolischen Grenzen des Labors als Lern- und Entwicklungsraum positionieren sich die $\mathrm{TN}$ als innovationsorientierte Mitarbeitende mit besonderen Kompetenzen in ihren entsendenden Organisationen. Zum anderen sollen die Innovationen Impulse für die entsendenden Organisationen bringen, die hier aus methodischen Gründen noch nicht untersucht werden konnten. Aus unserer Sicht birgt das untersuchte Projekt mit seinem umfangreichen Mixed-Methods-Materialzuschnitt insbesondere dann spannende Ergebnisse, wenn diese als verschränkte Gesamtergebnisse vorliegen.

Open Access Dieser Artikel wird unter der Creative Commons Namensnennung 4.0 International Lizenz (http://creativecommons.org/licenses/by/4.0/deed.de) veröffentlicht, welche die Nutzung, Vervielfältigung, Bearbeitung, Verbreitung und Wiedergabe in jeglichem Medium und Format erlaubt, sofern Sie den/die ursprünglichen Autor(en) und die Quelle ordnungsgemäß nennen, einen Link zur Creative Commons Lizenz beifügen und angeben, ob Änderungen vorgenommen wurden.

\section{Literatur}

Bacigalupo, M., Kampylis, P., Punie, Y., \& Van den Brande, G. (2016). EntreComp: The Entrepreneurship Competence Framework. Luxembourg: Publication Office of the European Union. https://doi.org/10. 2791/593884. EUR 27939 EN

Berdelmann, K. (2010). Operieren mit Zeit. Empirie und Theorie von Zeitstrukturen in Lehr-Lernprozessen. Paderborn: Schöningh.

Bueger, C. (2013). Pathways to practice: Praxiography and international politics. European Political Science Review. https://doi.org/10.1017/S1755773913000167.

Deakins, D., \& Freel, M. (1998). Entrepreneurial Learning and the Growth Process in SMEs. The Learning Organization, 5(3), 144-155.

Durst, S., \& Stahle, P. (2013). Success Factors of Open Innovation-A Literature Review. International Journal of Business Research and Management, 4(4), 111-131.

Göhlich, M. (1993). Die pädagogische Umgebung. Eine Geschichte des Schulraums seit dem Mittelalter. Weinheim: Deutscher Studienverlag.

Gryszkiewicz, L., Lykourentzou, I., \& Toivonen, T. (2016). Innovation Labs: Leveraging Openness for Radical Innovation? https://ssrn.com/abstract=2556692. Zugegriffen: 17. Febr. 2019. 
Hof, C., Kade, J., \& Fischer, M.E. (2010). Serielle Bildungsbiographien - Auf dem Weg zu einem qualitativen Bildungspanel zum Lebenslangen Lernen. Zeitschrift für Pädagogik, 56(3), 328-339.

Jochumsen, H., Skot-Hansen, D., \& Hvenegaard-Rasmussen, C. (2014). Erlebnis, Empowerment, Beteiligung und Innovation: Die neue Öffentliche Bibliothek. In O. Eigenbrodt \& R. Stang (Hrsg.), Formierungen von Wissensräumen. Berlin/Boston: De Gruyter Saur.

Jones, C., \& English, J. (2004). A contemporary approach to entrepreneurship education. Education + Training, 46, 416-423.

Kraus, K., Stang, R., Schreiber-Barsch, S., \& Bernhard, C. (2016). Erwachsenenbildung und Raum. Eine Einleitung. In K. Kraus, R. Stang, S. Schreiber-Barsch \& C. Bernhard (Hrsg.), Erwachsenenbildung und Raum. Theoretische Perspektiven, professionelles Handeln, Rahmungen des Lernens (S. 11-25). Bielefeld: wbv.

Lackéus, M. (2015). Entrepreneurship in Education. What, why, when, how. Paris: OECD.

Löw, M. (2001). Raumsoziologie. Frankfurt a.M.: Suhrkamp.

March, James G. (1991). Exploration and Exploitation in Organizational Learning. Organization Science, 2(1), 71-87.

Mayring, P. (2010). Qualitative Inhaltsanalyse: Grundlagen und Techniken (11. Aufl.). Weinheim: Beltz.

Mulgan, G. (2012). The Theoretical Foundations of Social Innovation. In A. Nickels \& A. Murdock (Hrsg.), Social Innovations (S. 33-65). London: Palgrave Macmillan.

Nandan, M., London, M., \& Bent-Goodley, T. (2015). Social Workers as Social Change Agents: Social Innovation, Social Intrapreneurship, and Social Entrepreneurship. Human Service Organizations: Management, Leadership \& Governance, 39(1), 38-56. https://doi.org/10.1080/23303131.2014.955236.

Osterwalder, A., \& Pigneur, Y. (2010). Business Model Generation: A Handbook for Visionaries, Game Changers, and Challengers. Hoboken, New Jersey: John Wiley \& Sons, Inc.

Plattner, H., Meinel, C., \& Weinberg, U. (2009). Design Thinking: Innovation lernen - Ideenwelten öffnen. München: mi-wirtschaftsbuch.

Prange, K. (2009). Zeit in Zeit. Über das Verhältnis von Unterrichtszeit und gelebter Zeit. In E. Protner (Hrsg.), Pädagogische Konzeptionen zwischen Vergangenheit und Zukunft (S. 43-52). Frankfurt a.M.: Peter Lang.

Reckwitz, A. (2002). Toward a Theory of Social Practices. A Development in Culturalist Theorizing. European Journal of Social Theory, 5(2), 243-263.

Rickards, T.J. (1994). Creativity from a business school perspective: Past, present and future. In S.G. Isaksen, M. C. Murdock, R. L. Firesien \& D. J. Treffinger (Hrsg.), Nurturing and developing creativity (S. 155-176). Norwood, NJ: Ablex.

Ries, E. (2011). The Lean Startup: How Today's Entrepreneurs Use Continuous Innovation to Create Radically Successful Businesses. New York: Crown Publishing Group.

Schatzki, T. R. (2001). Introduction: Practice theory. In T. R. Schatzki, K. D. Knorr-Cetina \& E. v. Savigny (Hrsg.), The Practice Turn in Contemporary Theory (S. 10-23). London and New York: Routledge.

Schmidt-Lauff, S. (2018). Zeittheoretische Implikationen in der Erwachsenenbildung. In R. Tippelt \& A. von Hippel (Hrsg.), Handbuch Erwachsenenbildung/Weiterbildung (S. 319-338). Wiesbaden: Springer.

Schmitz, B., \& Schröer, A. (2016). How Giants Learn to Dance. Towards Conceptualizing the Social Intrapreneur. Arbeitspapiere der Evangelischen Hochschule Darmstadt, Bd. 21.

Schröer, A. \& Händel, R.B. (2019). Social Intrapreneurship Labs - organisationspädagogische Grundlegung und empirische Befunde. In A. Schröer et al. (Hrsg.), Organisation und Zivilgesellschaft. (S. 269-280). Wiesbaden: Springer VS.

Schröer, A. \& Schmitz, B. (2019). Eine Methode zur Innovationsförderung in der Sozialwirtschaft. Das Labor für Diakonisches Unternehmertum (LaDU). In: B. Becher \& I. Hastedt (Hrsg.), Innovative Unternehmen in der Sozial- und Gesundheitswirtschaft (S. 143-170). Wiesbaden: Springer VS.

Schumpeter, J. (1942). Capitalism, Socialism, Democracy. New York: Routledge.

Swedberg, R. (2009). Schumpeter's full model of entrepreneurship: economic, non-economic and social entrepreneurship. In R. Ziegler (Hrsg.), An Introduction to Social Entrepreneurship (S. 77-106). Cheltenham: Elgar.

Zaltman, G., Duncan, R., \& Holbeck, J. (1973). Innovation and Organizations. New York: Wiley.

Publisher's Note Springer Nature remains neutral with regard to jurisdictional claims in published maps and institutional affiliations. 